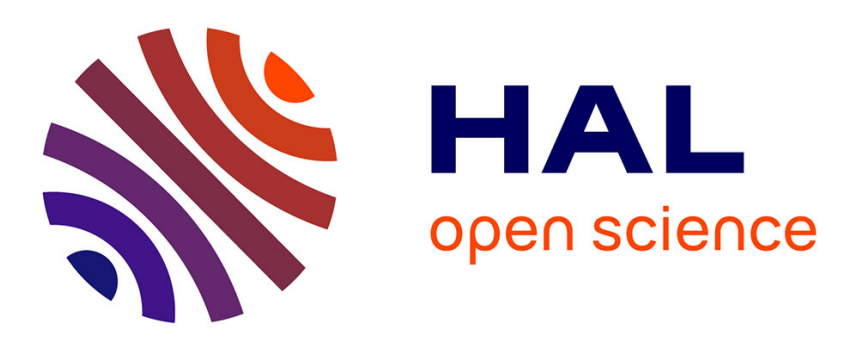

\title{
First-principles investigation of the bulk properties of americium dioxide and sesquioxides
}

Martin S. Talla Noutack, Grégory Geneste, Gérald Jomard, Michel Freyss

\section{To cite this version:}

Martin S. Talla Noutack, Grégory Geneste, Gérald Jomard, Michel Freyss. First-principles investigation of the bulk properties of americium dioxide and sesquioxides. Physical Review Materials, 2019, 3, pp.035001. 10.1103/PhysRevMaterials.3.035001 . cea-02063498

\section{HAL Id: cea-02063498 https://hal-cea.archives-ouvertes.fr/cea-02063498}

Submitted on 11 Mar 2019

HAL is a multi-disciplinary open access archive for the deposit and dissemination of scientific research documents, whether they are published or not. The documents may come from teaching and research institutions in France or abroad, or from public or private research centers.
L'archive ouverte pluridisciplinaire HAL, est destinée au dépôt et à la diffusion de documents scientifiques de niveau recherche, publiés ou non, émanant des établissements d'enseignement et de recherche français ou étrangers, des laboratoires publics ou privés. 


\title{
First-principles investigation of the bulk properties of americium dioxide and sesquioxides
}

\author{
Martin S. Talla Noutack, ${ }^{1}$ Grégory Geneste, ${ }^{2}$ Gérald Jomard, ${ }^{1}$ and Michel Freyss ${ }^{1, *}$ \\ ${ }^{1}$ CEA, DEN, DEC, Centre de Cadarache, 13108 Saint-Paul-lez-Durance, France \\ ${ }^{2}$ CEA, DAM, DIF, F-91297 Arpajon, France
}

(Received 18 May 2018; published 4 March 2019)

\begin{abstract}
We report an investigation of the ground-state properties of americium dioxide and its sesquioxides using first-principles calculations. In order to take into account strong $5 f$ electronic correlations, we apply the generalized gradient approximation $(\mathrm{GGA})+U$ as well as the hybrid functionals to these compounds. We present a systematic study of several bulk properties such as structural, elastic, energetic, electronic, and magnetic properties as a function of the effective Coulomb $U$ and exchange $J$ GGA $+U$ parameters in the range of $0.0-8.0$ and $0.00-0.75 \mathrm{eV}$, respectively. The values $(U, J)=(6.00 \mathrm{eV}, 0.75 \mathrm{eV})$ are those providing a correct description of bulk properties by comparison to the available experimental data. We discuss the effect of loss of symmetry caused by DFT $+U$ on several properties. We also discuss the effect of the spin-orbit coupling on these properties. Furthermore, we highlight that in order to reach the ground state of americium dioxide and sesquioxides using GGA $+U$ the monitoring of the occupation matrices of $5 f$ correlated orbitals is crucial. Our computational scheme provides reliable and accurate results concerning several bulk properties that have not been studied experimentally yet. For instance, our elastic constants calculated for $\mathrm{AmO}_{2}$ follow the same trend as those of other actinide oxides $\left(\mathrm{UO}_{2}, \mathrm{NpO}_{2}\right.$, and $\left.\mathrm{PuO}_{2}\right)$ and can be used as reference. We have also computed the electronic and static dielectric constants, as well as the Am and $\mathrm{O}$ Born effective charge of americium dioxide. Finally, we find the A-type hexagonal structure to be the most stable structure at low temperature for americium sesquioxides. For this phase, we predict the internal structural parameters as well as the bulk modulus. We provide data concerning the formation enthalpy of the $\mathrm{Am}_{2} \mathrm{O}_{3}$ bcc cubic structure.
\end{abstract}

DOI: 10.1103/PhysRevMaterials.3.035001

\section{INTRODUCTION}

Actinide compounds exhibit many interesting physical features owing to their partially filled $5 f$ orbitals $[1,2]$ and attract much interest for their industrial applications. In particular, americium oxides are considered as potential material for radioisotope thermoelectric generators for space exploration devices [3-5]. In addition, uranium-plutonium mixed oxide envisaged as the fuel for fast nuclear reactors will be fabricated from spent light water reactor fuel and will, as a consequence, contain a few percent of minor actinides (Am, $\mathrm{Cm}$, and $\mathrm{Np}$ ). One of current challenges is to determine whether the presence of minor actinides in low concentration, especially americium, will degrade properties of nuclear fuels for fast reactors.

Density functional theory (DFT) for actinide oxides is extremely challenging because of the complex features of the actinide $5 f$ electrons, which require dedicated treatment. Indeed, traditional exchange-correlation functionals like localdensity approximation and generalized gradient approximation (GGA) do not capture the strong electron-electron interaction due to the localization of the $f$ electrons.

In order to overcome this shortcoming, several approaches have been developed and have already been applied to americium dioxide $\left(\mathrm{AmO}_{2}\right)$ such as hybrid functionals [6,7], selfinteraction correction (SIC) [8,9], and intra-atomic Coulomb

\footnotetext{
*Corresponding author: michel.freyss@cea.fr
}

interaction using a Hubbard-type term $[4,10]$. The latter is the so-called DFT $+U$ approach, which has been extensively and successfully used for a wide range of correlated materials. The use of DFT $+U$ requires prior determination or calculation of the onsite Coulomb interaction $U$ and exchange $J$ parameters related to the Hubbard term. However, theoretical or experimental data on the properties of americium oxides are very scarce. It is thus important to first study fundamental properties of americium oxides.

Whereas the onsite Coulomb interaction $U$ and exchange $J$ are well known for $\mathrm{UO}_{2}[11,12]$, this is not the case for americium oxides, for which literature does not agree on any value since these values strongly vary from one author to another [7,13-15]. Actinide oxides have been extensively studied by the DFT $+U[12,16-18]$ method. Thus, we use the DFT $+U$ method in this paper and we purpose to provide the optimal values of the $U$ and $J$ parameters for americium oxides, which will be used in a subsequent study to evaluate the effect of americium in mixed actinide oxides. In this context, we also perform calculations using the hybrid functional to test the reliability of our $U$ and $J$ parameters.

Despite some studies reported in the literature, the knowledge of most bulk properties of americium oxides remains limited. To our knowledge, dielectric properties of americium oxides remain unknown. For instance, the static dielectric constant has never been either measured or calculated. Although the magnetic ground state of $\mathrm{UO}_{2}$ is identified as a transverse $3 \mathrm{k}$ antiferromagnetic (AFM) state below $30 \mathrm{~K}$ [19], the literature does not provide detailed information concerning $\mathrm{AmO}_{2}$. 
As regards americium sesquioxides $\left(\mathrm{Am}_{2} \mathrm{O}_{3}\right)$, the stable crystal structure at low temperature is not fully established [20]. In addition the only available studies on americium sesquioxides reported in literature aim at determining the lattice constants [21-23] and the band gap [21]. Thus, the $\mathrm{Am}_{2} \mathrm{O}_{3}$ compounds require a deeper study.

Furthermore, the literature reports only one study, carried out by Yong et al. [14], aimed at computing the elastic constants of $\mathrm{AmO}_{2}$ using the DFT $+U$ approximation. However, the bulk modulus obtained in this paper largely deviates from the experimental value. In addition, in a recent study published by Pegg and co-workers [13] using DFT $+U$ calculation, one can see that the evolution of the band gap as a function of the $U$ parameter is not smooth. The reason for these discrepancies is likely related to the fact that the system is trapped into metastable states during calculations $[24,25]$. The symmetry lowering associated with DFT $+U$ may cause an increase in the number of metastable states. Dorado et al. [12] showed that in $\mathrm{UO}_{2}$ the presence of metastable states induces large differences in the formation energy of point defects [16].

This paper reports results on the structural, elastic, magnetic, electronic, dielectric, and energetic properties of $\mathrm{AmO}_{2}$ and $\mathrm{Am}_{2} \mathrm{O}_{3}$ (cubic and hexagonal structures) in the framework of DFT $+U$ and the hybrid functional parametrized by Heyd-Scuseria-Ernzerhof (HSE) [3]. In Sec. II, we present the computational details. In Sec. III, we introduce the occupation matrices control for correlated orbitals applied to actinides oxides, which allows us to reach the ground state. In Sec. IV, we present our computed structural, elastic, magnetic, electronic, dielectric, and energetic properties in $\mathrm{AmO}_{2}$ yielded by the procedure presented in Sec. III. In Sec. V, we investigate the crystal structure stability of $\mathrm{Am}_{2} \mathrm{O}_{3}$ at low temperature using the same procedure to avoid metastable states. Finally, we present in this same section our results on the calculated ground-state properties of $\mathrm{Am}_{2} \mathrm{O}_{3}$ hexagonal and cubic structures.

\section{COMPUTATIONAL METHOD}

\section{A. Calculations parameters}

Our DFT calculations are carried out using both the Vienna Ab initio Simulation Package (VASP) [26,27] and the ABINIT package [28-30]. We use the projector augmented wave [31,32] formalism, which is particularly efficient for the description of complex phases in which atomic relaxations are important.

The exchange and correlation effects are described by the GGA parametrized by Perdew, Burke, and Ernzerhof (PBE) [33]. In order to take into account the strong correlations among $5 f$ electrons, an additional onsite Coulomb repulsion was considered by including a Hubbard-like term in the Hamiltonian [10]. The rotationally invariant form of Liechtenstein and Katsnelson [34] was used for the electron interaction energy from the Hubbard term $\left(E_{\mathrm{Hub}}\right)$. The total energy is the sum of the GGA energy ( $E^{\mathrm{GGA}}$ ) for a given density, the interaction term $E_{\mathrm{Hub}}$, and the double counting term $E_{\mathrm{dc}}$ :

$$
E^{\mathrm{GGA}+U}=E^{\mathrm{GGA}}+E_{\mathrm{Hub}}-E_{\mathrm{dc}} .
$$

The last two terms depend on the occupation matrix of the correlated orbitals.
For the double counting expression, we have chosen the "fully localized limit" (FLL) [10,35] because the ground state of americium oxides is insulating and thus orbitals occupation of $5 f$ electrons is close to one or zero.

Results are obtained using a plane-wave cutoff energy equals to $500 \mathrm{eV}$ using VASP code and $871 \mathrm{eV}$ using ABINIT code. According to our convergence tests, these input values lead to a precision lower than $1 \mathrm{meV}$ per atom. Unit cells containing 2 f.u. (6 atoms) as well as 4 f.u. (12 atoms) are used. For the 6-atom cell, one lattice vector $(c)$ is along one axis of the cubic fluorite structure, while the two others are turned by $45^{\circ}$ with respect to the cubic directions and have lengths corresponding to $\sqrt{2} / 2$ times the cubic lattice constant. Using VASP, the calculations are done on a $6 \times 6 \times 6$ k-points mesh generated by the Monkhorst-Pack [36] method, which is sufficient for an energy convergence less than $0.3 \mathrm{meV}$ per atom. With ABINIT, we used a $6 \times 6 \times 4$ k-point mesh. We performed full relaxation of the cell until the pressure acting on the system becomes lower than $0.01 \mathrm{kbar}$ (VASP) and $5 \times$ $10^{-7} \mathrm{Ha} / \mathrm{bohr}^{3}(\sim 0.15 \mathrm{kbar}$ ) (ABINIT), until the convergence on energy becomes less than $10^{-5} \mathrm{eV}$ per atom (VASP), and until the convergence on forces for the structural optimization becomes less than $5 \times 10^{-5} \mathrm{Ha} / \mathrm{bohr}(\sim 2.5 \mathrm{meV} / \AA$ ) (ABINIT). In order to ensure the convergence of calculations to the ground state, a practical occupation matrix control scheme for the $5 f$ correlated orbitals is used [12]. Note that VASP and ABINIT codes are simultaneously used in the whole paper (except in Sec. V, in which VASP has been exclusively used), and we ensure that the results obtained are in agreement. Sometimes, either the ABINIT or VASP code does not offer the possibility to compute certain properties. In this case we clearly indicate which one is used.

\section{B. Linear response calculations}

The electronic dielectric tensor and Born effective charges in $\mathrm{AmO}_{2}$ are calculated by using the density functional perturbation theory (DFPT) [37,38], as implemented in the ABINIT code. DFPT is a variational approach that allows computing the second derivatives of the total energy with respect to various perturbations, such as an external homogeneous electric field.

In an insulator, the Born effective charge tensor $Z_{K, \alpha \beta}^{*}$ of atom $K$ is the first derivative of the polarization component along direction $\beta$ with respect to the displacement of atom $K$ along direction $\alpha$. It can also be written as a mixed second derivative of the total energy with respect to such displacement and to the macroscopic electric field. The static dielectric tensor connects, at linear order, the macroscopic displacement field to the macroscopic electric field; it describes how an insulator is able to polarize under the application of an external electric field. It can be written as the second derivative of the total energy with respect to the electric-field components. The so-called relaxed-ion dielectric tensor contains two parts: first, the electronic dielectric tensor, which is the contribution due to the polarization of the electrons at fixed atomic positions; second, a sum of contributions due to atomic displacements, which can be here obtained from the knowledge of the phonon modes at the $\Gamma$ point. In this paper, the phonon modes at $\Gamma$ are obtained by using a frozen-phonon methodology (see Sec. IV B 4). 


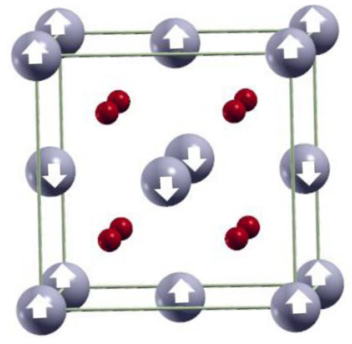

(a)

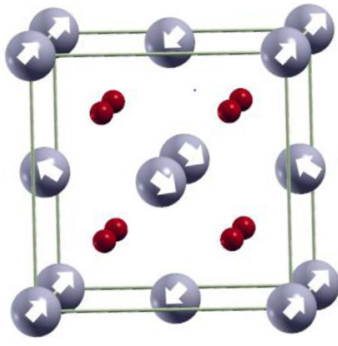

(b)
FIG. 1. $\mathrm{AmO}_{2}$ fluorite structure (12-atom cell): (a) longitudinal $1 \mathrm{k}$ collinear order and (b) transverse $3 \mathrm{k}$ noncollinear order. Arrows illustrate the $1 \mathrm{k}$ and $3 \mathrm{k}$ orientations of magnetic moments of spin carried by the americium atoms. Red atoms are oxygen atoms. $1 \mathrm{k}=$ [001] and $3 \mathrm{k}=[111] . J=0.75 \mathrm{eV}$ was used.

\section{GROUND STATE AND METASTABLE STATE OF AmO}

The use of the DFT $+U$ approximation induces an increase in the number of metastable states which makes the convergence to the ground state difficult. Such metastable states have been already reported in other compounds such as $\gamma$ and $\beta$ cerium [24], plutonium dioxide $\mathrm{PuO}_{2}$ [25], and uranium oxide $\mathrm{UO}_{2}$ [12]. It has been shown that in $\mathrm{UO}_{2}$ the presence of metastable states induces large differences in calculated properties, for instance in the formation energy of point defects [16]. Indeed, the DFT $+U$ approximation promotes the integer occupation of the correlated orbitals and, thereby, creates an anisotropy of the electronic density and thus of the crystal cell. The investigations by Jomard et al. [25] on plutonium oxides provide a practical scheme which consists in calculating and comparing the energies of all filling configurations of $5 f$ orbitals and therefore allows one to determine the ground state. The metastable states could be also expected in $\mathrm{AmO}_{2}$ using DFT $+U$ since it is a compound with strongly correlated $5 f$ orbitals. This section is dedicated to the calculation of the ground state of $\mathrm{AmO}_{2}$, considering collinear magnetism (i.e., the magnetization is a scalar quantity). An antiferromagnetic order similar to the $1 \mathrm{k}$ collinear order is imposed in the calculations [see Fig. 1(a)].

\section{A. Calculation taking symmetries into account}

In metallic americium the electronic configuration of the valence orbitals is $5 f^{7} 6 s^{2}$, and in americium dioxide the oxidation state of americium atoms is +IV with five electrons in the $5 f$ orbitals. There are $C_{7}^{5}=21$ different manners, called electronic configurations, to fill the seven $5 f$ orbitals of a given spin channel with five electrons, i.e., assuming for $\mathrm{Am}^{4+}$ a high-spin electronic configuration. As a consequence, only two $f$ orbitals of a given americium atom should be not filled. Our purpose in this section is to determine the ground-state occupation matrix of Am atoms in $\mathrm{AmO}_{2}$ using the occupation matrix control scheme.

Twenty-one diagonal matrices expressed in the basis of the real spherical harmonics, ordered as in Ref. [39], i.e., by increasing $m$, with the $x, y$, and $z$ directions being those of the conventional cell of the cubic fluorite structure, have been imposed at the beginning of each calculation. Each matrix corresponds to one particular electronic configuration of $5 f$
TABLE I. Relative energies per $\mathrm{AmO}_{2}$, lattice constant, magnetic moment per $\mathrm{Am}^{+4}$, and band gap of the states obtained starting from the 21 initial diagonal occupation matrices. The energy of the lowest state is set to zero. The results are given for $U=6 \mathrm{eV}$ and $J=0.75 \mathrm{eV}$. The lattice constant given corresponds to that of a cubic crystal that would have the same volume. The calculations were performed taking the symmetry of the crystal into account.

\begin{tabular}{lrrrc}
\hline \hline $\begin{array}{l}\text { Initial } \\
\text { matrix }\end{array}$ & $\begin{array}{r}\left(E-E_{\min }\right) / \mathrm{Am} \\
(\mathrm{meV})\end{array}$ & $\begin{array}{c}A \\
(\AA)\end{array}$ & $\begin{array}{c}\mu_{\mathrm{mag}} \\
\left(\mu_{B}\right)\end{array}$ & $\begin{array}{c}\text { Band gap } \\
(\mathrm{eV})\end{array}$ \\
\hline$[0011111]$ & 207.86 & 5.53 & 5.9 & 0.0 \\
{$[0101111]$} & 207.86 & 5.53 & 5.9 & 0.0 \\
{$[0110111]$} & 0.00 & 5.44 & 5.3 & 1.1 \\
{$[0111011]$} & 207.86 & 5.53 & 5.9 & 0.0 \\
{$[0111101]$} & 370.48 & 5.48 & 5.5 & 0.2 \\
{$[0111110]$} & 572.06 & 5.44 & 5.2 & 0.4 \\
{$[1001111]$} & 207.86 & 5.53 & 5.9 & 0.0 \\
{$[1010111]$} & 0.00 & 5.44 & 5.3 & 1.1 \\
{$[1011011]$} & 207.86 & 5.53 & 5.9 & 0.0 \\
{$[1011101]$} & 370.48 & 5.48 & 5.5 & 0.2 \\
{$[1011110]$} & 207.86 & 5.53 & 5.9 & 0.0 \\
{$[1100111]$} & 0.00 & 5.44 & 5.3 & 1.1 \\
{$[1101011]$} & 982.33 & 5.45 & 5.2 & 0.2 \\
{$[1101101]$} & 370.48 & 5.48 & 5.5 & 0.2 \\
{$[1101110]$} & 207.86 & 5.53 & 5.9 & 0.0 \\
{$[1110011]$} & 0.00 & 5.44 & 5.3 & 1.1 \\
{$[1110101]$} & 207.86 & 5.53 & 5.9 & 0.0 \\
{$[1110110]$} & 0.00 & 5.44 & 5.3 & 1.1 \\
{$[1111001]$} & 370.48 & 5.48 & 5.5 & 0.2 \\
{$[1111010]$} & 207.86 & 5.53 & 5.9 & 0.0 \\
{$[1111100]$} & 370.48 & 5.48 & 5.5 & 0.2 \\
\hline \hline
\end{tabular}

orbitals. Referring to the cubic crystal field, $f$ levels are partially degenerated. This suggests that some initial states should lead to the same final states. However, in order to check the efficiency of the method on the $\mathrm{AmO}_{2}$ system, we use these 21 occupation matrices as the starting point. We adopted the following notation for the occupation matrix:

$$
[1111100]=\left(\begin{array}{ccccccc}
1 & 0 & 0 & 0 & 0 & 0 & 0 \\
0 & 1 & 0 & 0 & 0 & 0 & 0 \\
0 & 0 & 1 & 0 & 0 & 0 & 0 \\
0 & 0 & 0 & 1 & 0 & 0 & 0 \\
0 & 0 & 0 & 0 & 1 & 0 & 0 \\
0 & 0 & 0 & 0 & 0 & 0 & 0 \\
0 & 0 & 0 & 0 & 0 & 0 & 0
\end{array}\right) .
$$

Each diagonal occupation matrix configuration is imposed during the first steps of the first self-consistent cycle. We first impose the crystal symmetry in our calculations (density, forces, and stress). Note that the use here of a $1 \mathrm{k}$ antiferromagnetic order changes the cubic $F m \overline{3} m$ space group to the tetragonal $P 4 / \mathrm{mmm}$ space group. We used several values of the $U$ parameter in the range of $4.00-8.00 \mathrm{eV}$ and two values of the $J$ parameter $(0.50$ and $0.75 \mathrm{eV})$. This choice will be justified in Sec. IV B 4. Depending on the initial matrix, several different states are reached. We present our results in Table I, setting the lowest energy to $0 \mathrm{eV}$. We decide to display in this table only the case in which the $U$ parameter equals $6 \mathrm{eV}$ and $J=0.75 \mathrm{eV}$. Indeed, for any value of $U$, 
the initial matrices leading to the lowest energy remain the same although the number of final states slowly increases when $U$ increases. The $J$ parameter does not have a significant influence on the final state.

We observe from Table I five different final states reached by the self-consistent procedure, which strongly depend on the initial occupation matrix. Actually, depending on the orbitals filled, the system is sometimes trapped in a local minimum and going to another minimum would require too much energy since the path from one minimum to another would involve partial occupancies. Out of these five states we have two metallic states and three insulating ones. Concerning the lowest-energy state, there are five initial matrices that finally lead to that state, so we can clearly conclude that we have five initial electronic configurations which allow us to reach the ground state. For this ground state, the occupation matrix for correlated electrons of the majority-spin channel is given by

$$
M^{\text {sym }}=\left(\begin{array}{lllllll}
1.02 & 0.00 & 0.00 & 0.00 & 0.00 & 0.00 & 0.00 \\
0.00 & 0.32 & 0.00 & 0.00 & 0.00 & 0.00 & 0.00 \\
0.00 & 0.00 & 1.03 & 0.00 & 0.00 & 0.00 & 0.00 \\
0.00 & 0.00 & 0.00 & 0.10 & 0.00 & 0.00 & 0.00 \\
0.00 & 0.00 & 0.00 & 0.00 & 1.03 & 0.00 & 0.00 \\
0.00 & 0.00 & 0.00 & 0.00 & 0.00 & 1.02 & 0.00 \\
0.00 & 0.00 & 0.00 & 0.00 & 0.00 & 0.00 & 1.02
\end{array}\right)
$$

\section{B. Calculation without taking symmetries into account}

In this section, we are now interested in the influence of the crystal symmetries on the ground state. DFT $+U$ is likely to break the cubic symmetry and then may induce after structural relaxation a slight difference between the lattice constants $a, b$, and $c$. It is then important to evaluate the effect of the reduction of the symmetry number on the ground-state properties of $\mathrm{AmO}_{2}$. Thus, we perform our calculations without taking any symmetry into account using the ABINIT code. We use $U=6 \mathrm{eV}$ and $J=0.75 \mathrm{eV}$. We have performed two searches.

(1) The lattice constants and atomic positions are fixed (in the perfect cubic geometry) and the 21 matrices are tested (imposed over the 20 first steps of the electronic loop). The final matrix corresponding to the lowest energy is then

$$
M_{1}^{\text {no sym }}=\left(\begin{array}{rrrrrrr}
0.44 & 0.00 & -0.42 & 0.00 & 0.00 & 0.00 & 0.00 \\
0.00 & 0.25 & 0.00 & 0.00 & 0.00 & 0.00 & 0.00 \\
-0.42 & 0.00 & 0.66 & 0.00 & 0.00 & 0.00 & 0.00 \\
0.00 & 0.00 & 0.00 & 0.99 & 0.00 & 0.00 & 0.00 \\
0.00 & 0.00 & 0.00 & 0.00 & 0.99 & 0.00 & 0.00 \\
0.00 & 0.00 & 0.00 & 0.00 & 0.00 & 0.99 & 0.00 \\
0.00 & 0.00 & 0.00 & 0.00 & 0.00 & 0.00 & 0.99
\end{array}\right) .
$$

Another state has an energy only $1 \mathrm{meV}$ above, with a matrix $M_{2}^{\text {no sym }}$ very close to that found in the symmetric case:

$$
M_{2}^{\text {no sym }}=\left(\begin{array}{ccccccc}
0.99 & 0.00 & 0.00 & 0.00 & 0.00 & 0.00 & 0.00 \\
0.00 & 0.25 & 0.00 & 0.00 & 0.00 & 0.00 & 0.00 \\
0.00 & 0.00 & 0.99 & 0.00 & 0.00 & 0.00 & 0.00 \\
0.00 & 0.00 & 0.00 & 0.12 & 0.00 & 0.00 & 0.00 \\
0.00 & 0.00 & 0.00 & 0.00 & 0.99 & 0.00 & 0.00 \\
0.00 & 0.00 & 0.00 & 0.00 & 0.00 & 0.99 & 0.00 \\
0.00 & 0.00 & 0.00 & 0.00 & 0.00 & 0.00 & 0.99
\end{array}\right) .
$$

(2) The previous calculations are extended by a full structural optimization (cell and atomic positions). At the end, the configurations corresponding to $M_{1}^{\text {no sym }}$ and $M_{2}^{\text {no sym }}$ have their energy slightly lowered, but the matrices are preserved all along the relaxation. However, we observe that another configuration jumps during the relaxation onto a different electronic state, leading to a final configuration that is more stable than the ones corresponding to $M_{1}^{\text {no sym }}$ and $M_{2}^{\text {no sym }}$ by $\sim 0.08-0.09 \mathrm{eV}^{-} \mathrm{AmO}_{2}$. 
The final matrix of that case is

$$
M_{3}^{\text {no sym }}=\left(\begin{array}{rrrrrrr}
0.80 & 0.01 & -0.15 & 0.22 & -0.15 & 0.00 & 0.19 \\
0.01 & 0.25 & 0.01 & -0.00 & 0.01 & -0.00 & -0.01 \\
-0.15 & 0.01 & 0.88 & 0.17 & -0.11 & 0.00 & 0.15 \\
0.22 & -0.00 & 0.17 & 0.73 & 0.17 & 0.00 & -0.22 \\
-0.15 & 0.00 & -0.11 & 0.17 & 0.88 & -0.01 & 0.15 \\
0.00 & -0.00 & 0.01 & 0.00 & -0.00 & 0.99 & 0.00 \\
0.19 & -0.00 & 0.15 & -0.22 & 0.15 & 0.00 & 0.80
\end{array}\right) .
$$

Table II displays the results obtained at the end of the structural optimization. We have 15 different final states: the number of metastable states has significantly increased with respect to the search of the previous section in which symmetries are accounted for. This is probably a consequence of symmetry breaking. Furthermore, the lowest energy obtained is roughly $90 \mathrm{meV}$ lower than the one obtained when the symmetries are taken into account. Moreover, the matrix very close to that of the symmetric case $\left(M^{\mathrm{sym}}\right)$ corresponds here to the third metastable state. It is clear that the reduction of the number of symmetries increases the probability of the system to be trapped into a metastable state during the calculation.

Finally, we observe that, for $M_{1}^{\text {no sym }}, M_{2}^{\text {no sym }}$, and $M_{3}^{\text {no sym }}$, the $\mathrm{Am}^{4+}$ magnetic moment is almost the same as in the symmetric case. Nevertheless, we observe a slight difference

TABLE II. Relative energies $\mathrm{AmO}_{2}$, lattice constant, and $\mathrm{Am}^{4+}$ magnetic moment of the states obtained starting from the 21 initial diagonal occupation matrices. The energy of the lowest state is set to zero. The lattice constant given corresponds to that of a cubic crystal that would have the same volume. The calculations were performed without taking any symmetry into account.

\begin{tabular}{lcccc}
\hline \hline $\begin{array}{l}\text { Initial } \\
\text { matrix }\end{array}$ & $\begin{array}{c}\left(E-E_{\min }\right) / \mathrm{Am} \\
(\mathrm{eV})\end{array}$ & $\begin{array}{c}A \\
(\AA)\end{array}$ & $\begin{array}{c}\mu_{\mathrm{mag}} \\
\left(\mu_{B}\right)\end{array}$ & $\begin{array}{c}\text { Occupation } \\
\text { matrix }\end{array}$ \\
\hline$[0011111]$ & 0.08 & 5.44 & 5.2 & $M_{1}^{\text {no sym }}$ \\
{$[0101111]$} & 0.85 & 5.49 & 5.2 & \\
{$[0110111]$} & 0.76 & 5.46 & 5.1 & $M_{3}^{\text {no sym }}$ \\
{$[0111011]$} & 0.00 & 5.44 & 5.2 & \\
{$[0111101]$} & 0.95 & 5.46 & 5.9 & \\
{$[0111110]$} & 0.32 & 5.49 & 5.6 & $M_{1}^{\text {no sym }}$ \\
{$[1001111]$} & 0.08 & 5.44 & 5.2 & $M^{\text {sym }}$ \\
{$[1010111]$} & 0.09 & 5.44 & 5.2 & $M_{2}^{\text {no sym }} \sim .4$ \\
{$[1011011]$} & 0.08 & 5.44 & 5.2 & \\
{$[1011101]$} & 0.64 & 5.47 & 5.4 & $M_{1}^{\text {no sym }}$ \\
{$[1011110]$} & 0.08 & 5.44 & 5.2 & \\
{$[1100111]$} & 0.75 & 5.47 & 5.1 & \\
{$[1101011]$} & 1.24 & 5.48 & 5.1 & \\
{$[1101101]$} & 0.95 & 5.46 & 5.9 & \\
{$[1101110]$} & 0.77 & 5.48 & 5.2 & \\
{$[1110011]$} & 0.75 & 5.47 & 5.2 & \\
{$[1110101]$} & 0.66 & 5.48 & & \\
{$[1110110]$} & 0.76 & 5.46 & 5.1 & \\
{$[1111001]$} & 0.47 & 5.45 & & \\
{$[1111010]$} & 0.61 & 5.50 & 5.4 & \\
{$[1111100]$} & 0.95 & 5.46 & 5.9 & \\
\hline \hline
\end{tabular}

between the lattice constants $(a \neq b \neq c)$ but the cell volume $(a \times b \times c)$ remains in the three cases very close to the case where crystal symmetry is taken into account. Finally, the band gaps are in the three cases rather close to each other around $1.1( \pm 0.2) \mathrm{eV}$. Therefore, the calculations taking into account crystal symmetry are expected not to have a significant effect on the bulk properties.

\section{BULK PROPERTIES OF $\mathrm{AmO}_{2}$}

Knowing the $5 f$ occupation matrix of the ground state of the $\mathrm{AmO}_{2}$ system, it is possible to correctly model bulk properties. In order to evaluate these properties using DFT $+U$, it is essential to determine the values of the onsite Coulomb interaction $U$ and the exchange $J$ parameters for $\mathrm{AmO}_{2}$. Kotani et al. [11] proposed a systematic analysis of actinide $4 f$ core $\mathrm{x}$-ray photoemission spectra in the actinide dioxide series using the Anderson impurity model. Based on the electronic states of these materials they determined the $U$ and $J$ parameters. For $\mathrm{AmO}_{2}$, they find $U=6.00 \mathrm{eV}$ and $J=$ $0.75 \mathrm{eV}$. At present, there is no explicit calculation of these parameters in order to validate the Kotani values. As a result, the literature does not agree on any value of $U$ and $J$, since the values used in the early first-principles study strongly vary from one author to another [7,13-15].

Our investigation in this section aims at determining the values of onsite Coulomb parameters allowing us to correctly describe the bulk properties using the GGA $+U$ approximation as parametrized by PBE [33]. We evaluate structural properties, electronic properties, elastic properties, enthalpy of formation, magnetic properties including or not spinorbit coupling (SOC) as a function of $U$ and $J$ parameters, as well as dielectric properties. $U$ varies in the range of $0.0-8.0 \mathrm{eV}$, and $J$ varies in the range of $0.0-0.75 \mathrm{eV}$. The $5 f$ electrons occupation matrix $\left(M^{\mathrm{sym}}\right)$ for the symmetric case obtained in Sec. III A 1 has been used to avoid the metastable states. Finally, we determine the parameters that best reproduce experimentally known properties. In order to test the consistency and reliability of our studies, we also perform calculation using the hybrid functional approximation as parametrized by Heyd-Scuseria-Ernzerhof [6]. Indeed, this functional does not require prior knowledge of onsite Coulomb interaction $U$ and exchange $J$ parameters but the ratio of Hartree-Fock exchange (generally set at 25\%) used in this approximation. However, the computing time is extremely large compared to DFT $+U$ calculations. Our results have been 

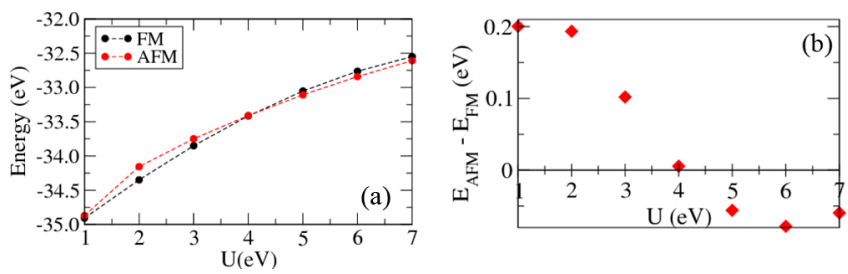

FIG. 2. (a) Total energy as a function of the $U$ parameter. Black circles correspond to the ferromagnetic order while red circles correspond to the $1 \mathrm{k}$ antiferromagnetic order. (b) Energy difference between AFM and FM ordering with respect to $U$. VASP code was used here.

compared to other available HSE results as well as experimental results.

\section{A. Magnetic structure of $\mathrm{AmO}_{2}$}

According to the experimental results [40,41], the magnetic ground state of $\mathrm{AmO}_{2}$ is known to be antiferromagnetic. Furthermore, our calculation using the HSE functional shows that the antiferromagnetic order is more stable than the ferromagnetic (FM) order. In fact, we find the relative energy $E_{\mathrm{AFM}}-E_{\mathrm{FM}}$ equals to $-0.03 \mathrm{eV}$ per $\mathrm{AmO}_{2}$ formula (see Table IV). In order to model $\mathrm{AmO}_{2}$ using GGA $+U$ calculation, we compute the energies of the ferromagnetic and antiferromagnetic orders for several $U$ values. We plot in Fig. 2(a) the total energy of AFM and FM ordering per unit formula as a function of the $U$ parameter. This figure is completed by displaying the energy difference between these magnetics orders [see Fig. 2(b)]. We can see that the values of the $U$ parameter larger than $4 \mathrm{eV}$ lead to negative values of the $E_{\mathrm{AFM}}-E_{\mathrm{FM}}$ difference and thus offer a good description of the magnetic ground state.

Although the magnetic ground state of $\mathrm{AmO}_{2}$ is known to be an antiferromagnetic state, the literature does not provide any explicit investigation on the type (collinear or noncollinear) of the AFM order (see Fig. 1). In $\mathrm{UO}_{2}$ the magnetic ground state is identified as a transverse 3k AFM state [19]. We here study the relative stability between longitudinal $1 \mathrm{k}$ and transverse $3 \mathrm{k}$ AFM orders including the spin-orbit coupling and we present our results in Fig. 3 and Table III. Figure 3(a) shows that $1 \mathrm{k}$ AFM order is the most stable order starting from the value $U=4 \mathrm{eV}$ when the SOC is not included in calculations. Conversely, Fig. 3(b) shows that
TABLE III. Lattice parameter, $c / a$ ratio, difference between the energies of $1 \mathrm{k}$ AFM order and 3k AFM order, and spin magnetic moment in $\mathrm{AmO}_{2}$ depending on different orientations of spin magnetic moments ( $1 \mathrm{k}$ and $3 \mathrm{k}$ ) without and with SOC. We used $U=6 \mathrm{eV}$ and $J=0.75 \mathrm{eV}$ in DFT $+U$ calculations. The occupation matrix used is the one determined in Sec. III A.

\begin{tabular}{lcccc}
\hline \hline & $\begin{array}{c}a \\
(\AA)\end{array}$ & $c / a$ & $\begin{array}{c}\mu_{\mathrm{mag}} \\
\left(\mu_{B}\right)\end{array}$ & $\begin{array}{c}\Delta E=E-E_{1 \mathrm{k} \text { or } 1 \mathrm{k}+\mathrm{SOC}} \\
(\mathrm{eV})\end{array}$ \\
\hline 1k order & 5.44 & 0.99 & 5.3 & 0.00 \\
1k order + SOC & 5.46 & 1.00 & 2.2 & 0.00 \\
3k order & 5.51 & 1.00 & 5.8 & 0.29 \\
3k order +SOC & 5.50 & 1.00 & 2.4 & -0.10 \\
Exp. & $5.37^{\mathrm{a}}$ & 1.00 & $1.4^{\mathrm{b}}$ & \\
\hline \hline
\end{tabular}

${ }^{\text {a Reference [23]. }}$

${ }^{\mathrm{b}}$ Reference [41].

$3 \mathrm{k}$ AFM order is more stable than the $1 \mathrm{k}$ order with an energy difference equal to $0.1 \mathrm{eV}$ per unit formula when the contribution of the SOC is added.

The investigation by Tokunaga et al. [42] on $\left(\mathrm{Pu}_{0.91} \mathrm{Am}_{0.09}\right) \mathrm{O}_{2}$ using ${ }^{17} \mathrm{O}$ nuclear magnetic resonance (NMR) shows an effective magnetic moment of the $1.38 \mu_{B} / \mathrm{Am}^{4+}$ ion around $5.8 \mathrm{~K}$. Our computed magnetic moment $\left(2.2\right.$ or $\left.2.4 \mu_{B}\right)$ when spin-orbit coupling is included is not close to this experimental result but is nevertheless much closer than the one obtained without taking SOC into account. In addition, we observe that $3 \mathrm{k}$ AFM order tends to increase the cell volume (Table III). This can be attributed to the distortion occurring in the oxygen sublattice. In fact, we observe the oxygen sublattice distortion of roughly $0.17 \AA$ along the crystallographic directions $\langle 111\rangle$. Our result confirms the observations made by Tokunaga et al. [42]. Indeed, from the frequency curve with respect to temperature, they observe a NMR line broadening and they suppose that this NMR line broadening could arise from oxygen sublattice distortions. Based on these results, we suggest that the $\mathrm{AmO}_{2}$ magnetic ground state is a transverse 3k AFM order with an oxygen sublattice distortion of roughly $0.17 \AA$. However, the longitudinal 1k AFM order without SOC remains a reasonable approximation of the magnetic ground state of $\mathrm{AmO}_{2}$ (3k AFM order) for the computing of bulk properties although the magnetic moment is poorly described $\left(5.3 \mu_{B}\right.$ instead of $\left.1.4 \mu_{B}\right)$.
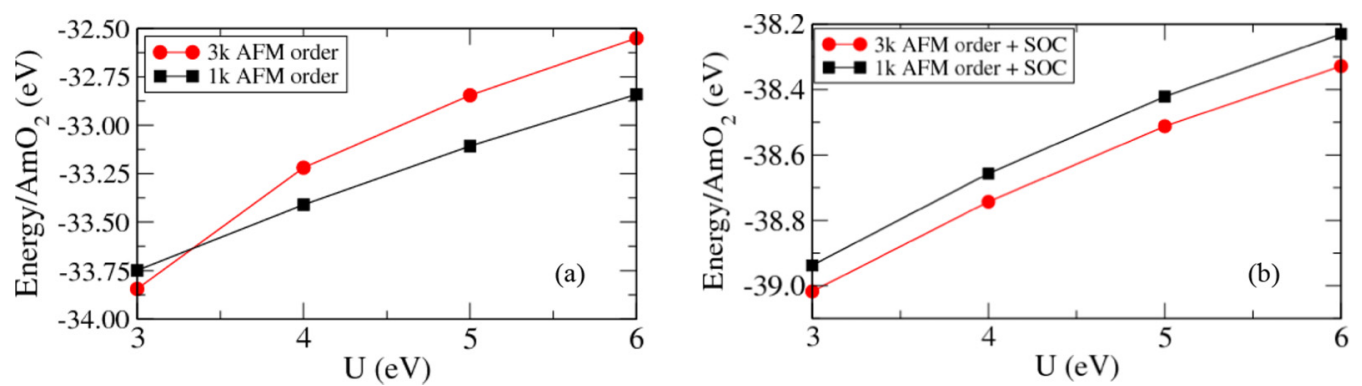

FIG. 3. Total energy per americium atom as a function of the $U$ parameter. The red line is $3 \mathrm{k}$ antiferromagnetic order while the black line is $1 \mathrm{k}$ antiferromagnetic order. (a) SOC is not included and (b) SOC is included. Calculations were performed on $\mathrm{AmO}_{2}$ fluorite structure (12-atom cell) using VASP code. 


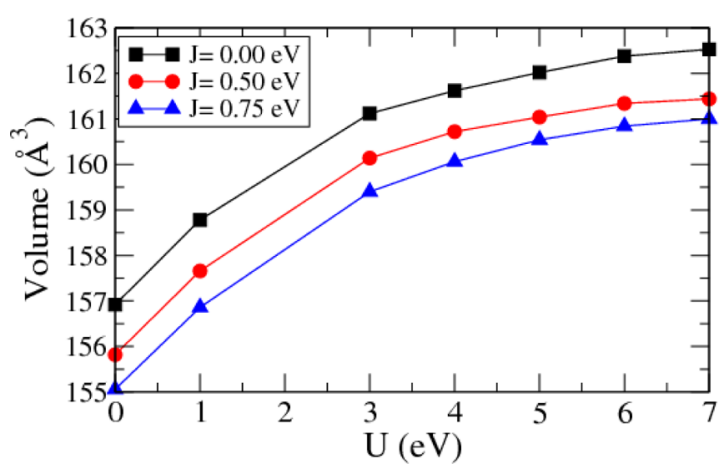

FIG. 4. Evolution of the cell volume as a function of the $U$ and $J$ parameters.

\section{B. Other bulk properties of $\mathrm{AmO}_{2}$}

Modeling the $3 \mathrm{k}$ AFM order including SOC requires a considerable computing time in a large supercell. Moreover, another challenge here would be to determine the ground-state occupation matrix allowing modeling of the $3 \mathrm{k}$ AFM order including SOC. We thus come back to collinear magnetism and use longitudinal 1k AFM order without taking into account SOC to model $\mathrm{AmO}_{2}$ since we showed in Sec. IV A that, without including SOC, the 1k AFM order is more stable than the transverse $3 \mathrm{k}$ AFM order and can be used as a good approximation of the $\mathrm{AmO}_{2}$ magnetic ground state. The investigations in $\mathrm{AmO}_{2}$ (using the hybrid functional) by Wen et al. [7] or in $\mathrm{UO}_{2}[12,18]$ and in $\mathrm{PuO}_{2}$ [25] also show that $1 \mathrm{k}$ AFM order can be used as an approximation of the $3 \mathrm{k}$ AFM order to successfully compute bulk properties. Nevertheless, it is important to note that $1 \mathrm{k}$ AFM approximation changes the point group $\mathrm{O}_{h}$ to $D_{4 h}$. For instance, one can clearly see (Table III) that the $c / a$ ratio differs from 1 . In this case, the crystalline field splits the $5 f$ orbitals in two twofolddegenerate levels $\left(2 \times E_{u}\right)$ and three nondegenerate levels
$\left(\mathrm{A}_{1 u}, \mathrm{~B}_{1 u}\right.$, and $\left.\mathrm{B}_{2 u}\right)$. Except when explicitly mentioned, the calculations are now performed using the matrix obtained by taking into account symmetries.

\section{Structural properties}

In this part, we compute the lattice constant using different values of the $U$ and $J$ parameters. We then evaluate the influence of the $J$ parameter on the cell volume in order to choose the $J$ value that allows us to obtain the volume closest to the experimental one $\left(\sim 155 \AA^{3}\right)$. We observe a slight decrease of the volume when the $J$ parameter increases (see Fig. 4). The value $J=0.75 \mathrm{eV}$ leads to the smallest volume, and we keep it in all the rest of our calculations. From Table IV, we can see that the computed lattice constant $(a=$ $5.36 \AA$ ) obtained with HSE approximation is very close both to the experimental value ( $a=5.37 \AA$ ) and to other calculated values like the one obtained by Wen et al. [7] using HSE $(a=5.38 \AA)$. Our GGA $+U$ computed value $(a=5.44 \AA)$ is slightly above the experimental data $(1.3 \%)$ but is still in a reasonable agreement. The same trend is observed for other computed values available in literature (see Table IV).

\section{Electronic properties}

In Fig. 5(a), we present the band gap calculated as a function of the $U$ parameter. Our calculated band gap $(1.1 \mathrm{eV})$ using $\mathrm{GGA}+U(6 \mathrm{eV})$ is close to the experimental one $(1.3$ $\mathrm{eV}$ ) though it is slightly underestimated (see Table IV). We see that the band gap obtained with the HSE06 functional $(1.6 \mathrm{eV})$ is overestimated but is in agreement with those obtained by Wen et al. [7] using the same functional. We also present in Fig. 5(b) the electronic density of states projected onto the Am $f$ spin-up and spin-down states and the $\mathrm{O} p$ orbitals in order to provide a more detailed description of the electronics properties of $\mathrm{AmO}_{2}$ (the calculation is performed here without symmetry, using the $M_{3}^{\text {no sym }}$ matrix). We set the top of the valence-band maximum as the energy reference.

TABLE IV. Calculated lattice parameter, band gap, bulk modulus, elastic constants, formation enthalpy, Am ${ }^{4+}$ magnetic moment, and relative stability of magnetic order. For comparisons, previous experimental results as well as other theoretical results are listed. Since there is no experimental value of elastic constants, the ones of $\mathrm{UO}_{2}$ and $\mathrm{PuO}_{2}$ are also listed in order to show a trend. In parenthesis: formation enthalpy estimated using a total energy for $\mathrm{O}_{2}$ equal to $2 E_{\text {tot }}(\mathrm{O})+E_{\text {bind }}\left(\mathrm{O}_{2}\right)$, where $E_{\text {tot }}(\mathrm{O})$ is the total energy of the $\mathrm{O}$ atom (spin polarized, no symmetry) and $E_{\text {bind }}\left(\mathrm{O}_{2}\right)$ is the experimental binding energy of the $\mathrm{O}_{2}$ molecule.

\begin{tabular}{|c|c|c|c|c|c|c|c|c|c|c|}
\hline Comp. & Method & $a(\AA)$ & Gap $(\mathrm{eV})$ & $\mathrm{C}_{11}(\mathrm{GPa})$ & $\mathrm{C}_{12}(\mathrm{GPa})$ & $\mathrm{C}_{44}(\mathrm{GPa})$ & $B_{0}(\mathrm{GPa})$ & $\Delta H_{f}(\mathrm{eV})$ & $\mu_{\mathrm{mag}}\left(\mu_{B}\right)$ & $E_{\mathrm{AFM}}-E_{\mathrm{FM}} / \mathrm{Am}(\mathrm{eV})$ \\
\hline & $\mathrm{GGA}+U(5 \mathrm{eV})$ & 5.44 & 0.7 & 361 & 101 & 73 & 187 & $\begin{array}{l}-8.56 \\
(-9.42)\end{array}$ & 5.3 & -0.05 \\
\hline & $\mathrm{GGA}+U(6 \mathrm{eV})$ & 5.44 & 1.1 & 363 & 102 & 71 & 189 & $\begin{array}{l}-8.29 \\
(-9.15)\end{array}$ & 5.3 & -0.06 \\
\hline \multirow[t]{7}{*}{$\mathrm{AmO}_{2}$} & HSE06 & 5.36 & 1.6 & - & - & - & 210 & - & 5.1 & -0.03 \\
\hline & $\mathrm{GGA}+U^{\mathrm{a}}$ & 5.44 & - & - & - & - & - & - & - & -0.03 \\
\hline & $\mathrm{GGA}+U^{\mathrm{b}}(4 \mathrm{eV})$ & 5.43 & 0.0 & - & - & - & - & - & - & - \\
\hline & $\mathrm{GGA}+U^{\mathrm{c}}(4 \mathrm{eV})$ & 5.35 & 1.0 & 204 & 87 & 55 & 126 & - & 4.77 & - \\
\hline & $\mathrm{SIC}^{\mathrm{d}}$ & 5.42 & 0.8 & - & - & - & 209 & - & - & - \\
\hline & $\mathrm{HSE}^{\mathrm{b}}$ & 5.38 & 1.5 & - & - & - & - & - & - & - \\
\hline & Exp. & $5.37^{\mathrm{e}}$ & $1.3^{\mathrm{a}}$ & - & - & - & $205^{\mathrm{f}}$ & $-9.51^{\mathrm{g}}$ & - & $\mathrm{AFM}^{\mathrm{h}}$ \\
\hline $\mathrm{UO}_{2}$ & Exp. & - & - & $389^{i}$ & $119^{i}$ & $60^{i}$ & $207^{\mathrm{f}}$ & - & - & - \\
\hline $\mathrm{PuO}_{2}$ & $\mathrm{GGA}+U^{\mathrm{j}}$ & - & - & 375 & 111 & 70 & 178 & - & - & - \\
\hline
\end{tabular}

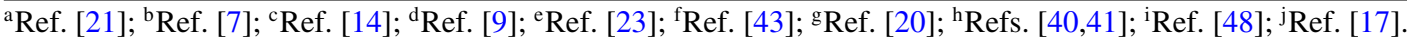



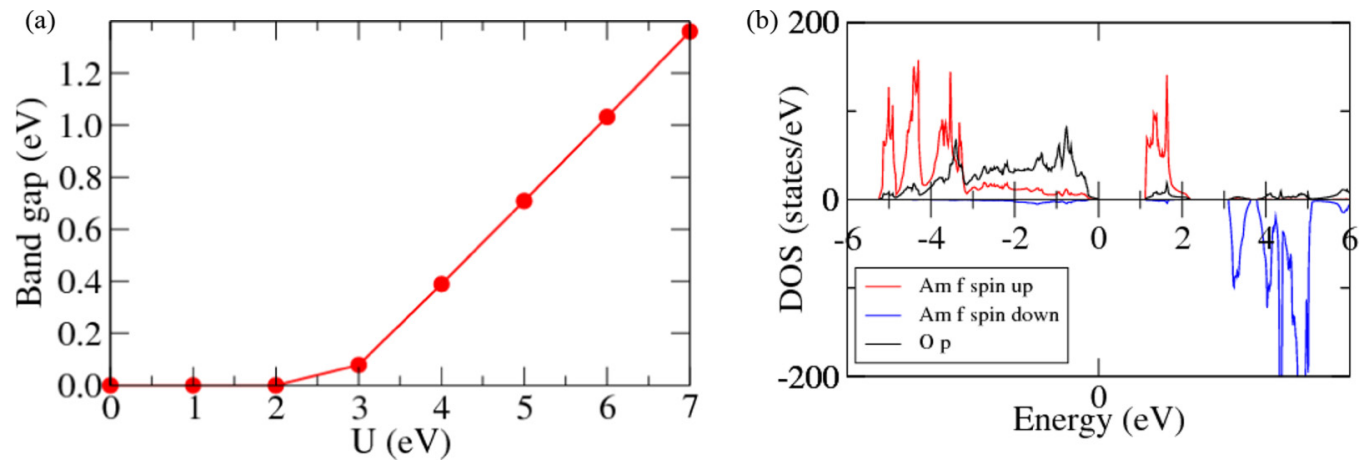

FIG. 5. (a) Evolution of the band gap as a function of the $U$ parameter. (b) Electronic density of states (DOS) projected onto the Am $f$ (spin up and spin down) and $\mathrm{O} p$ orbitals in $\mathrm{AmO}_{2}$.

The results obtained show that the band gap of $\mathrm{AmO}_{2}$ is mostly formed between the $\mathrm{O} p$ occupied bands and the Am $f$ unoccupied bands. We can conclude that $\mathrm{AmO}_{2}$ is charge transfer insulating from the $\mathrm{O} p$ to Am $f$ components.

\section{Elastic properties}

Up to now, no experimental study aimed at determining elastic constants of $\mathrm{AmO}_{2}$ exists to our knowledge and the theoretical studies are scarce. The only available study in the literature has been performed by Yong et al. [14] using the $\mathrm{GGA}+U$ approximation. However, the bulk modulus obtained from the elastic constants in this paper largely deviates from the experimental value. The metastable states could explain the origin of the discrepancies observed in the results obtained by Yong et al. Figure 6 represents the evolution of the elastic constants as a function of the $U$ parameter. The results obtained show the same trend as those obtained in $\mathrm{UO}_{2}, \mathrm{NpO}_{2}$, and $\mathrm{PuO}_{2}$ (see Table IV) and can be regarded as reference values. We observe that $\mathrm{C}_{11}$ and $\mathrm{C}_{12}$ remain almost constant with the increase of $U$ while $C_{44}$ slightly decreases. The bulk modulus values obtained from these values are less than $10 \%$ off the experimental value [43] (Table IV), which is acceptable. This small underestimation is within the error expected for the computational method $(\mathrm{GGA}+U)$. Our calculated value $(210 \mathrm{GPa})$ using the HSE06 functional is close to the experimental value (205 GPa) [43]

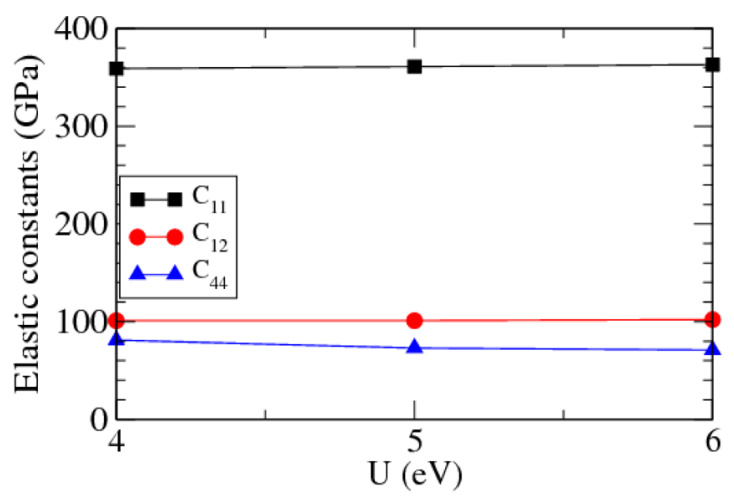

FIG. 6. Evolution of the elastic constants with respect to the $U$ parameter. Calculations were performed on $\mathrm{AmO}_{2}$ fluorite structure (12-atom cell) using VASP code. and the one obtained by Petit et al. (209 GPa) using the SIC method [9].

Although the results (lattice constant and bulk modulus) obtained with the HSE06 functional are closer to the experimental results than the GGA $+U$ ones, the HSE06 functional requires much longer computing time compared to the GGA $+U$ method. Nevertheless, a good agreement with the experimental results as well as other HSE06 results allows us to conclude on the reliability of our paper.

\section{Dielectric properties}

To our knowledge, dielectric properties of americium oxides remain unknown. For instance, the static dielectric constant has never been either measured or calculated. Using DFPT as implemented in the ABINIT code, we have performed response function calculations to obtain the electronic dielectric tensor and the Born effective charges. The calculations are performed using the 6-atom unit cell, (i) taking into account the symmetries (case 1) and (ii) without symmetry (case 2) (the occupation matrix and cell vectors are the ones corresponding to the $M^{\text {sym }}$ matrix in the first case and to the $M_{3}{ }^{\text {nosym }}$ matrix in the second case).

The phonon modes at the $\Gamma$ point of the first Brillouin zone have been obtained using a finite-difference (frozenphonon) scheme: the atoms are displaced one by one along each direction, by $+\delta$ and $-\delta$ (with $\delta=0.0025$ a.u.), and the total energy and atomic forces of the distorted configuration are recomputed, which allows us to construct the force constant matrix and then the dynamical matrix at $\Gamma$. The latter is diagonalized, providing the phonon pulsations $\omega_{i}{ }^{2}$, and phonon eigenvectors, from which the mode effective charge and the oscillator strength tensor are constructed for each of the 18 modes. Each mode possessing a nonzero effective charge contributes to the static dielectric constant [44]. These contributions are then computed and added to the electronic dielectric tensor to obtain the (relaxed-ion) static dielectric tensor. Convergence with $\delta$ is checked by doing the same calculation using $2 \delta$ (which provides the same dielectric constant with a precision of 0.01 ). We still use $U=6 \mathrm{eV}$, $J=0.75 \mathrm{eV}$.

We obtain the following results, where small deviations from diagonal tensors (in case 2) are only due to the loss of symmetry caused by DFT $+U$. 
a. Born effective charges. In case 1,

$$
\begin{aligned}
Z_{\mathrm{Am}}^{*} & =\left(\begin{array}{lll}
5.14 & 0.00 & 0.00 \\
0.00 & 5.14 & 0.00 \\
0.00 & 0.00 & 4.58
\end{array}\right), \\
Z_{\mathrm{O}}^{*} & =\left(\begin{array}{rrr}
-2.57 & 0.00 & 0.00 \\
0.00 & -2.57 & 0.00 \\
0.00 & 0.00 & -2.29
\end{array}\right) .
\end{aligned}
$$

In case 2 ,

$$
\begin{aligned}
Z_{\mathrm{Am}}^{*} & =\left(\begin{array}{lll}
4.92 & 0.19 & 0.18 \\
0.19 & 4.92 & 0.18 \\
0.19 & 0.19 & 4.91
\end{array}\right), \\
Z_{\mathrm{O}}^{*} & =\left(\begin{array}{lll}
-2.46 & -0.10 & -0.09 \\
-0.10 & -2.46 & -0.09 \\
-0.09 & -0.09 & -2.46
\end{array}\right) .
\end{aligned}
$$

Small differences do exist between the two cases, due to different symmetries, but the tensors have the same trace: $1 / 3 \operatorname{Tr}\left(Z_{\mathrm{Am}}^{*}\right)=4.95$ (case 1) and $4.92($ case 2$) ; 1 / 3 \operatorname{Tr}\left(Z_{\mathrm{O}}^{*}\right)=$ -2.48 (case 1 ) and -2.46 (case 2).

b. Electronic dielectric tensor. In case 1,

$$
\epsilon^{\infty}=\left(\begin{array}{lll}
6.31 & 0.00 & 0.00 \\
0.00 & 6.31 & 0.00 \\
0.00 & 0.00 & 5.91
\end{array}\right) .
$$

In case 2,

$$
\epsilon^{\infty}=\left(\begin{array}{lll}
6.15 & 0.25 & 0.23 \\
0.25 & 6.15 & 0.23 \\
0.23 & 0.23 & 6.19
\end{array}\right) .
$$

Here again, both tensors have a very close trace: $1 / 3 \operatorname{Tr}(\varepsilon)=$ 6.17 (case 1) and 6.16 (case 2).

c. (Relaxed-ion) static dielectric tensor (thus including ionic contributions). In case 1 ,

$$
\epsilon_{S}=\left(\begin{array}{ccc}
19.18 & 0.0 & 0.0 \\
0.0 & 19.18 & 0.0 \\
0.0 & 0.0 & 13.83
\end{array}\right) .
$$

In case 2 ,

$$
\epsilon_{S}=\left(\begin{array}{ccc}
16.83 & 1.35 & 1.42 \\
1.35 & 16.82 & 1.42 \\
1.42 & 1.42 & 16.81
\end{array}\right) .
$$

The static dielectric constant is thus $1 / 3 \operatorname{Tr}\left(\varepsilon_{S}\right)=17.40$ (case 1) and 16.82 (case 2).

Knowing the experimental static dielectric constants of $\mathrm{UO}_{2}$ (23.8) [45] and $\mathrm{PuO}_{2}$ (18.9) [46], our value can be regarded as a reference since it follows the same trend.

We observe that the loss of symmetry caused by DFT $+U$ induces an anisotropy in the dielectric properties, and this anisotropy may be different from one occupation matrix to another. But it is satisfactory to see that the tensors obtained by the two occupation matrices have almost the same trace; that is to say, the dielectric properties averaged over the
Cartesian directions are preserved from one occupation matrix to another.

\section{Formation enthalpy of $\mathrm{AmO}_{2}$}

Finally, we also compute the enthalpy of formation $\Delta H_{f}$ of $\mathrm{AmO}_{2}$ with respect to molecular oxygen and metallic Am- $\alpha$ (computed using GGA $+U$ with the same $U$ and $J$ parameters in order to keep the same energy reference). This energy, related to the $\mathrm{Am}+\mathrm{O}_{2} \rightarrow \mathrm{AmO}_{2}$ reaction, can be written as follows:

$$
\Delta H_{f}^{\mathrm{AmO}_{2}}=E_{\mathrm{tot}}^{\mathrm{AmO}_{2}}-E_{\mathrm{tot}}^{\mathrm{Am}}-E_{\mathrm{tot}}^{\mathrm{O}_{2}},
$$

where $E_{\mathrm{tot}}^{\mathrm{AmO}_{2}}, E_{\mathrm{tot}}^{\mathrm{Am}}$, and $E_{\mathrm{tot}}^{\mathrm{O}_{2}}$ are the total energy of the $\mathrm{AmO}_{2}$ compound, of molecular oxygen, and of Am- $\alpha$. To our knowledge, there is only one experimental study [20] aimed at evaluating the formation enthalpy of $\mathrm{AmO}_{2}$. Our computed values $(-8.56$ and $-8.29 \mathrm{eV}$ with $U=5$ and $6 \mathrm{eV}$, respectively) deviate from the experimental value $(-9.51 \mathrm{eV})$ by roughly $10 \%$. The GGA approximation overestimates the cohesion energy of the $\mathrm{O}_{2}$ molecule $(-6.01 \mathrm{eV}$ instead of $-5.21 \mathrm{eV}$ as in Ref. [47]), which could explain part of the error obtained calculating cohesion and formation energies of oxide compounds within GGA $+U$ approximation. Another contribution of the $10 \%$ disagreement with the experiment could arise from the use of the Hubbard term to describe metallic americium. We then performed a correction in the energy of the oxygen molecule using the following approximation: $2 E_{\text {tot }}(\mathrm{O})+E_{\text {bind }}\left(\mathrm{O}_{2}\right)$, where $E_{\text {tot }}(\mathrm{O})$ is the total energy of the $\mathrm{O}$ atom (spin polarized, no symmetry) and $E_{\text {bind }}\left(\mathrm{O}_{2}\right)$ is the experimental [47] binding energy of the $\mathrm{O}_{2}$ molecule. This contributes to improve the enthalpy of formation, the new values being -9.42 and $-9.15 \mathrm{eV}$ with $U=5$ and $6 \mathrm{eV}$, respectively. One observes that the error with respect to the experimental value has decreased.

\section{Discussion on onsite Coulomb parameters $(U, J)$}

We summarize in Table IV all our computed results obtained on the bulk properties of $\mathrm{AmO}_{2}$. Regarding Sec. III A, the values of $U$ less than $5 \mathrm{eV}$ do not offer a good description of the magnetic ground state as experimentally known. The comparison of our results obtained with $\mathrm{GGA}+U(6 \mathrm{eV})$ to other computed values, on the one hand, and to the experimental results on the other hand shows a good agreement. Thus, we can clearly conclude that $(U, J)=(6 \mathrm{eV}, 0.75 \mathrm{eV})$ can be used to correctly model $\mathrm{AmO}_{2}$ using $\mathrm{GGA}+U$ as parametrized by PBE. This result confirms the values determined by Kotani et al. Indeed, the Kotani values have never been used in previous studies. The current paper thus provides the onsite Coulomb interaction $U$ and exchange $J$ by fitting on several bulk properties of $\mathrm{AmO}_{2}$. These values could be used to further study properties like formation and migration energies of point defect in a large defective supercell using GGA $+U$ calculations. Such properties are extremely important for nuclear application and other domains such as semiconductor physics. 


\section{INVESTIGATION OF PROPERTIES OF AMERICIUM SESQUIOXIDES $\left(\mathrm{Am}_{2} \mathrm{O}_{3}\right)$}

According to the experimental results, americium is reduced to $\mathrm{Am}^{3+}$ within mixed oxides such as $(\mathrm{U}, \mathrm{Am}) \mathrm{O}_{2}$ and (U, Pu, Am) $\mathrm{O}_{2}[22,49,50]$. In order to investigate the mixed oxides containing americium atoms, it is important to know some bulk properties of $\mathrm{Am}_{2} \mathrm{O}_{3}$. The investigation of americium sesquioxides remains up to now very scarce and the only available studies on americium sesquioxides reported in literature aim at determining the lattice constants [21-23] and the band gap [21]. In this part, we first investigate the stable crystal structure at low temperature, then we predict several bulk properties such as band gap, bulk modulus, structural parameters, formation enthalpy, and spin magnetic moment.

\section{A. Stable crystal structure of $\mathrm{Am}_{2} \mathrm{O}_{3}$ at low temperature}

From plutonium, the actinide sesquioxides (and also many other sesquioxides) crystallize into three different crystalline phases. These are the bcc C-type, monoclinic B-type, and hexagonal A-type. The stable crystal structure of americium sesquioxide at room temperature is not fully established. Early studies [51,52] report $\mathrm{Am}_{2} \mathrm{O}_{3}$ as a bcc C-type structure with space group $I a \overline{3}$ at room temperature. However, since $\mathrm{Pu}_{2} \mathrm{O}_{3}$ has a hexagonal A-type structure at room temperature and $\mathrm{Cm}_{2} \mathrm{O}_{3}$ has a monoclinic B-type structure, the bcc C-type structure at room temperature for $\mathrm{Am}_{2} \mathrm{O}_{3}$ is unlikely according to Konings et al. [20]. Furthermore, no oxygen potential measurements are fully consistent with the data of Sari and Zamorani previously mentioned [52]. Our purpose here is to investigate the americium sesquioxide, namely, bcc C-type and hexagonal A-type, and determine the stable structure of the crystal at low temperature. All experimental studies on the B-type are in agreement with the fact that it is stable only at high temperature [51-53], and, as a result, we will not investigate this phase.

Since $\mathrm{Am}_{2} \mathrm{O}_{3}$ contains correlated $5 f$ orbitals, it is essential to monitor the electronic occupations of these orbitals using DFT $+U$ as shown for $\mathrm{AmO}_{2}$. In $\mathrm{Am}_{2} \mathrm{O}_{3}, \mathrm{Am}^{3+}$ ions have six electrons in the $5 f$ orbitals. There are $C_{6}^{7}=7$ different configurations (still assuming high-spin electronic configurations) and therefore seven possible initial diagonal occupation matrices. We imposed these seven occupation matrices at the beginning of each calculation. We used $1 \mathrm{k}$ antiferromagnetic order [20] and $(U, J)=(6 \mathrm{eV}, 0.75 \mathrm{eV})$ as well as $(5 \mathrm{eV}$, $0.75 \mathrm{eV}$ ). The symmetry of the wave function has been taken into account. We display our results in Table V. For the hexagonal structure four different states are reached, including one metallic state.

As regards the cubic structure, five different final states depending of the initial matrix are reached with two metallic states (see Table V).

Knowing the ground states of bcc C-type and hexagonal Atype, we can now compare the total energy of these structures. The relative energy per $\mathrm{Am}_{2} \mathrm{O}_{3}$ formula unit is $E_{\mathrm{Cub}}-E_{\mathrm{Hex}}=$ $0.86 \mathrm{eV}$ (using $U=6 \mathrm{eV}$ ) and $0.80 \mathrm{eV}$ (using $U=5 \mathrm{eV}$ ), indicating that the hexagonal A-type is the most stable crystal structure of americium sesquioxide at low temperature. This result is not in agreement with the first thermodynamic phase
TABLE V. Relative energies obtained starting from the seven initial diagonal occupation matrices. The energy of the lowest state is set to zero. For bcc C-type, a 80 -atom supercell $(2 \times 2 \times 2)$ is used and a 5 -atom cell is used for the hexagonal A-type.

\begin{tabular}{lcc}
\hline \hline & \multicolumn{2}{c}{$\left(E-E_{\min }\right) / \mathrm{Am}(\mathrm{eV})$} \\
\cline { 2 - 3 } Initial matrix & A-type & C-type \\
\hline$[0111111]$ & 0.00 & 0.00 \\
$[101111]]$ & 0.00 & 0.00 \\
{$[1101111]$} & 0.00 & 0.53 \\
{$[1110111]$} & 0.00 & 0.00 \\
{$[1111011]$} & 1.25 & 0.33 \\
{$[1111101]$} & 0.66 & 0.23 \\
{$[1111110]$} & 0.31 & 0.21 \\
\hline \hline
\end{tabular}

diagram on the Am-O system reported by Sari and Zamorani [52] using differential thermal enthalpy. However, it is in agreement with the one reported by Gotcu-Freis et al. [54] using the CALPHAD method [55], though this CALPHAD phase diagram is not completely established owing to the lack of experimental data on the stochiometric C-type. More specifically, the existence of another stoichiometric sesquioxide phase with bcc structure reported by Sari and Zamorani [52] is still controversial. Furthermore, most experimental [46] and theoretical [20] studies on $\mathrm{Am}_{2} \mathrm{O}_{3}$ have been made on the hexagonal structure. Therefore, further experimental work is required to fully establish thermodynamic description of the Am-O system.

\section{B. Bulk properties of $\mathrm{Am}_{2} \mathrm{O}_{3}$}

Several bulk properties such as lattice parameters, band gap, magnetic moment, bulk modulus, and formation enthalpy of A-type and C-type structures have been calculated in this section. The computed internal structural parameters for Atype obtained after relaxation of all the degrees of freedom are $z_{\mathrm{Am}}=0.2448$ and $z_{0}=0.6504$. These parameters are found not to depend on the value of $U$ and are required to model hexagonal $\mathrm{Am}_{2} \mathrm{O}_{3}$ structure. Since there are neither experimental nor theoretical values up to now, our values can be used as reference. Table VI presents the results computed in this paper compared to the experimental results as well as the theoretical ones. Regarding the hexagonal $\mathrm{Am}_{2} \mathrm{O}_{3} \mathrm{~A}$-type, we can see that our computed lattice parameters show a good agreement with the experimental results yielded by Nishi et al. [22]. The lattices constants of bcc C-type obtained in this paper are slightly overestimated (1.4\%) compared to the experimental results obtained by Hurtgen and Fuger [23] but are nevertheless within the error expected for the computational method $(\mathrm{GGA}+U)$.

Our computed band gap $(2.85 \mathrm{eV})$ using GGA $+U(5 \mathrm{eV})$ is close to the only available theoretical result [21] $(2.60 \mathrm{eV})$ whereas the one computed using $\mathrm{GGA}+U(6 \mathrm{eV})$ shows a large discrepancy. Since the value obtained using the HSE functional $(2.2 \mathrm{eV})$ is close to one obtained with $\mathrm{GGA}+U$ ( $5 \mathrm{eV}$ ), it is likely that the $U=6 \mathrm{eV}$ value is not an appropriate value for $\mathrm{A}-\mathrm{Am}_{2} \mathrm{O}_{3}$. However, we cannot conclude because of the lack of additional data. For bcc C-type, the 
TABLE VI. Calculated lattice parameters, band gap, magnetic moment, and bulk modulus $\left(B_{0}\right)$ of both hexagonal A-type and bcc C-type $\mathrm{Am}_{2} \mathrm{O}_{3}$ structure. These properties are compared to experimental available data as well as theoretical ones. In parenthesis: formation enthalpy estimated using a total energy for $\mathrm{O}_{2}$ equal to $2 E_{\text {tot }}(\mathrm{O})+E_{\text {bind }}\left(\mathrm{O}_{2}\right)$, where $E_{\text {tot }}(\mathrm{O})$ is the total energy of the $\mathrm{O}$ atom (spin polarized, no symmetry) and $E_{\text {bind }}\left(\mathrm{O}_{2}\right)$ is the experimental binding energy of the $\mathrm{O}_{2}$ molecule.

\begin{tabular}{|c|c|c|c|c|c|c|c|c|}
\hline Comp & Method & $a(\AA)$ & $b(\AA)$ & $c(\AA)$ & Gap (eV) & $\mu_{\mathrm{mag}}\left(\mu_{B}\right)$ & $B_{0}(\mathrm{GPa})$ & $\Delta H_{f}(\mathrm{eV})$ \\
\hline \multirow{5}{*}{ A-type } & $\mathrm{GGA}+\mathrm{U}(5 \mathrm{eV})$ & 3.87 & 3.87 & 5.96 & 2.85 & 6.1 & 139 & $-16.04(-17.33)$ \\
\hline & $\mathrm{GGA}+\mathrm{U}(6 \mathrm{eV})$ & 3.87 & 3.87 & 5.98 & 3.29 & 6.2 & 139 & $-15.91(-17.20)$ \\
\hline & HSE06 & 3.77 & 3.77 & 6.04 & 2.2 & 5.9 & & \\
\hline & $\mathrm{GGA}+U^{\mathrm{a}}$ & & & & 2.60 & & & \\
\hline & Exp. ${ }^{b}$ & 3.82 & 3.82 & 5.98 & & & & $-17.52^{\mathrm{d}}$ \\
\hline \multirow{3}{*}{ C-type } & $\mathrm{GGA}+U(5 \mathrm{eV})$ & 11.17 & 11.17 & 11.21 & 1.17 & 6.1 & 100 & $-15.24(-16.53)$ \\
\hline & $\mathrm{GGG}+U(6 \mathrm{eV})$ & 11.17 & 11.17 & 11.21 & 1.25 & 6.2 & 135 & $-15.06(-16.35)$ \\
\hline & Exp. $^{\mathrm{c}}$ & 11.02 & 11.02 & 11.02 & & & & \\
\hline
\end{tabular}

${ }^{\mathrm{a}}$ Reference [21].

${ }^{\mathrm{b}}$ Reference [22].

${ }^{\mathrm{c}}$ Reference [23].

${ }^{\mathrm{d}}$ Reference [20].

evolution of the $U$ value does not have a significant influence on the band gap.

Concerning the bulk modulus, our calculated values, $135 \mathrm{GPa}$ for C-type and $139 \mathrm{GPa}$ for B-type, follow the same trend as the ones obtained in $\mathrm{Pu}_{2} \mathrm{O}_{3}$ (137-164 GPa) using $\mathrm{DFT}+U$ [17]. Concerning the A-type structure the bulk modulus remains the same when $U$ varies. The bulk moduli have never been either calculated or experimentally determined in $\mathrm{Am}_{2} \mathrm{O}_{3}$ C-type or A-type structure, and our values are then a prediction of $\mathrm{Am}_{2} \mathrm{O}_{3}$ bulk moduli. Indeed, owing to the good agreement of our computed lattice constants with the experimental data we are also confident on the reliability of the calculated bulk moduli.

Like for $\mathrm{AmO}_{2}$, we compute the $\mathrm{Am}_{2} \mathrm{O}_{3}$ formation enthalpies of both A-type and C-type structures, i.e., the energy related to the $2 \mathrm{Am}+3 / 2 \mathrm{O}_{2} \rightarrow \mathrm{Am}_{2} \mathrm{O}_{3}$ reaction (see Table VI). For the A-type, we observe that the correction applied in the energy of the $\mathrm{O}_{2}$ molecule allows us to obtain an energy roughly less than $5 \%$ off the experimental data [20]. For C-type structure, our computed value $-16.53 \mathrm{eV} \pm 1 \%$ constitutes a prediction (where $1 \%$ represents the relative error attributed to the $\mathrm{GGA}+U$ method compared to the experimental data, assuming that this error is almost the same as in $\mathrm{A}-\mathrm{Am}_{2} \mathrm{O}_{3}$ or $\mathrm{AmO}_{2}$ ). This value is one of the data on C-type needed for the CALPHAD method [53] to better refine the phase diagram of the Am-O system. Indeed, for the moment, this phase is not included in the CALPHAD model of the Am-O system due to the lack of data [53].

\section{CONCLUSION}

In this paper, we report results yielded by DFT $+U$ calculations on the ground-state properties of americium dioxide and its sesquioxides. Our paper contributes to establish fundamental bulk properties of americium oxides either unknown up to now or poorly known. We highlight that the presence of metastable states in DFT $+U$ calculations can account for the discrepancies observed in the literature for $\mathrm{AmO}_{2}$ regarding the elastic constants as well as the evolution of the band gap as a function of the $U$ parameter of the DFT $+U$. We show that the ground state can be reached by imposing occupation matrices at the beginning of calculations. We observe a slight difference between the lattice constants $(a \neq b \neq c)$ when crystal symmetry is not taken into account but the cell volume remains very close to the case in which the crystal symmetry is taken into account. Moreover, in both cases, the $\mathrm{Am}^{4+}$ magnetic moments are very close to each other, as are the band gaps $(1.1 \pm 0.2 \mathrm{eV})$. Finally, the density of states and the isotropic contributions to the dielectric properties (trace of Born effective charge and dielectric tensors) are unchanged by the loss of symmetry.

We compute several bulk properties of $\mathrm{AmO}_{2}$ and $\mathrm{Am}_{2} \mathrm{O}_{3}$ such as structural, elastic, energetic, electronic, and magnetic properties as a function of $U$ and $J$ parameters, as well as dielectric properties. Our results show a good agreement with the available data (experimental and computed). For $\mathrm{AmO}_{2}$, the values $(U, J)=(6.00 \mathrm{eV}, 0.75 \mathrm{eV})$ are those providing the best description of bulk properties by comparison to the available experimental data. We provide several bulk properties such as magnetic and elastic (elastic constants) properties, as well as the static dielectric constant of americium dioxide.

Concerning americium sesquioxides, we find the hexagonal A-type to be the most stable structure at low temperature. This result is the first theoretical confirmation (in agreement with the CALPHAD calculations reported by Gotcu-Freis et al. [54]) of the stability at low temperature of the hexagonal A-type structure of $\mathrm{Am}_{2} \mathrm{O}_{3}$, the phase diagram of which is still controversial. Furthermore, the structural properties computed in this paper are very close to the experimental results. We provide the internal structural parameters for the hexagonal structure, which can be used as reference for future works. We also predict the bulk modulus of both bcc C-type cubic and A-type hexagonal structures as well as the band gap and the formation enthalpy of the bcc C-type. We highlight that the $U=6 \mathrm{eV}$ and $J=0.75 \mathrm{eV}$ values probably tend to overestimate the band gap but do not affect other bulk properties.

The first-principles study proposed in this paper paves the way for an optimal use of the GGA $+U$ approximation to study 
$\mathrm{AmO}_{2}$ defective supercells and could be extended to study more complex materials like $(\mathrm{U}, \mathrm{Am}) \mathrm{O}_{2}$ mixed oxides.

\section{ACKNOWLEDGMENTS}

This work was performed using high-performance computing resources from Grand Equipement National de Calcul Intensif (GENCI) [Centre Commun de Recherche et de Technologie (CCRT) and Centre Informatique National de l'Enseignement Supérieur (CINES)]. This research is part of the Investigations Supporting MOX Fuel Licensing in ESNII Prototype Reactors (INSPYRE). We would like to thank I. Cheik Njifon for careful reading of the paper. We are also grateful to B. Amadon for fruitful discussions. Finally, L. Baguet and M. Torrent are acknowledged for their help with $\mathrm{DFPT}+U$.
[1] S. Heathmean, R. G. Haire, T. L. Bihan, A. Lindbaum, M. Idiri, P. Normile, S. Li, and G. H. Lander, Science 309, 110 (2005).

[2] R. Atta-Fynn and A. K. Ray, Phys. Rev. B 76, 115101 (2007).

[3] L. Summerer and K. Stephenson, Proceedings of the Institution of Mechanical Engineers, Part G: Journal of Aerospace Engineering (Sage Publications, London, UK, 2011), Vol. 225 , p. 129.

[4] R. C. O'Brien, R. Ambrosi, N. P. Bannister, S. D. Howe, and H. V. Atkinson, J. Nucl. Mater. 377, 506 (2008).

[5] T. Tinsley, M. Sarsfield, and T. Rice, Br. Interplanet. Spc. 64, 375 (2002).

[6] J. Heyd, G. E. Scuseria, and M. Ernzerhof, J. Chem. Phys. 105, 8207 (2003).

[7] X. D. Wen, R. L. Martin, L. E. Roy, G. E. Scuseria, S. P. Rudin, E. R. Batista, T. M. McCleskey, B. L. Scott, E. Bauer, J. J. Joyce, and T. Durakiewicz, J. Chem. Phys. 137, 154707 (2012).

[8] J. P. Perdew and A. Zunger, Phys. Rev. B 23, 5048 (1981).

[9] L. Petit, A. Svane, Z. Szotek, W. M. Temmerman, and G. M. Stocks, Phys. Rev. B 81, 045108 (2010).

[10] V. I. Anisimov, J. Zaanen, and O. K. Andersen, Phys. Rev. B 44, 943 (1991).

[11] A. Kotani and H. Ogasawara, Phys. B: Condens. Matter 186-188, 16 (1993)

[12] B. Dorado, B. Amadon, M. Freyss, and M. Bertolus, Phys. Rev. B 79, 235125 (2009)

[13] J. T. Pegg, X. Aparicio-Anglès, M. Storr, and N. H. de Leeuw, J. Nucl. Mater. 492, 269 (2017).

[14] L. Yong, Y. Yu, F. Zheng, B. T. Wanga, and P. Zhang, J. Nucl. Mater. 441, 411 (2013).

[15] A. Bendjedid, T. Seddik, R. Khenata, H. Baltache, G. Murtaza, A. Bouhemadou, S. B. Omran, S. Azam, and S. A. Khan, J. Magn. Mater 396, 190 (2015).

[16] B. Dorado, G. Jomard, M. Freyss, and M. Bertolus, Phys. Rev. B 82, 035114 (2010)

[17] I. C. Njifon, M. Bertolus, R. Hayn, and M. Freyss, Inorg. Chem. 57, 10974 (2018).

[18] E. Vathonne, Etude par calcul de structure électronique des dégâts d'irradiation dans le comsbustible nucléaire $\mathrm{UO}_{2}$ : Comportement des défauts ponctuels et gaz de fission, Ph.D. thesis, Aix-Marseille University, 2014.

[19] S. B. Wilkins, R. Caciuffo, C. Detlefts, J. Rebizant, E. Colineau, F. Wastin, and G. H. Lander, Phys. Rev. B 73, 060406(R) (2006).

[20] R. J. M. Konings, O. Beneš, A. Kovács, D. Manara, D. Sedmidubský, L. Gorokhov, V. S. Iorish, V. Yungman, E. Shenyavskaya, and E. Osina, J. Phys. Chem. Ref. Data 43, 013101 (2014).
[21] C. Suzuki, T. Nishi, M. Nakada, M. Akabori, M. Hirata, and Y. Kaji, J. Phys. Chem. Solids 73, 209 (2012).

[22] T. Nishi, M. Nakada, C. Suzuki, H. Shibata, A. Itoh, M. Akabori, and M. Hirata, J. Nucl. Mater. 401, 138 (2010).

[23] C. Hurtgen and J. Fuger, Inorg. Nucl. Chem. Lett. 13, 179 (1977).

[24] B. Amadon, F. Jollet, and M. Torrent, Phys. Rev. B 77, 155104 (2008).

[25] G. Jomard, B. Amadon, F. Bottin, and M. Torrent, Phys. Rev. B 78, 075125 (2008).

[26] G. Kresse and J. Hafner, Phys. Rev. B 47, 558(R) (1993).

[27] G. Kresse and J. Furthmüller, Comput. Mater. Sci. 6, 15 (1996).

[28] X. Gonze, J.-M. Beuken, R. Caraca, F. Detrau, M. Fuchs, G.-M. Rignanese, L. Sindi, M. Verstraet, G. Zerah, F. Jollet, M. Torrent, A. Roy, M. Mikami, P. Ghose, J.-Y. Raty, and D. Allan, Comput. Mater. Sci. 25, 478 (2002).

[29] X. Gonze, G.-M. Rignanese, M. Verstraete, Y. Pouillon, R. Caracas, F. Jollet, M. Torrent, G. Zerah, M. Mikami, P. Ghosez, M. Veithen, J.-Y. Raty, V. Olevano, F. Bruneval et al., Z. Kristallogr. 220, 558 (2005).

[30] The present results have been obtained through the use of the ABINIT code, a common project of the Université Catholique de Louvain; Corning, Inc.; and other contributors, see http://www. abinit.org.

[31] G. Kresse and D. Joubert, Phys. Rev. B 59, 1758 (1999).

[32] P. E. Blöch, Phys. Rev. B 50, 17953 (1994).

[33] J. P. Perdew, K. Burke, and M. Ernzerhof, Phys. Rev. Lett. 77, 3865 (1996).

[34] A. I. Lichtenstein and M. I. Katsnelson, Phys. Rev. B 57, 6884 (1998).

[35] A. I. Liechtenstein, V. I. Anisimov, and J. Zaanen, Phys. Rev. B 52, R5467(R) (1995).

[36] H. J. Monkhorst and J. D. Pack, Phys. Rev. B 13, 5188 (1976).

[37] X. Gonze, Phys. Rev. B 55, 10337 (1997).

[38] X. Gonze and C. Lee, Phys. Rev. B 55, 10355 (1997).

[39] M. A. Blancoa, M. Floreza, and M. Bermejo, J. Mol. Struct. 419, 19 (1997).

[40] D. G. Karraker, J. Chem. Phys. 63, 3174 (1975).

[41] Y. Tokunaga, T. Nishi, S. Kambe, M. Nakada, Y. Homma, H Sakai, and H. Chudo, J. Phys. Soc. Jpn. 79, 053705 (2010).

[42] Y. Tokunaga, M. Oaka, S. Kambe, S. Miwa, H. Sakai, H. Chudo, Y. Homma, and Y. Shiokawa, J. Nucl. Mater. 396, 107 (2010).

[43] M. Idiri, T. Le Bihan, S. Heathman, and J. Rebizant, Phys. Rev. B 70, 014113 (2004).

[44] E. Cockayn and B. P. Burton, Phys. Rev. B 62, 3735 (2000).

[45] N. Hampton, G. Saunders, J. Harding, and A. Stoneham, J. Nucl. Mater. 149, 18 (1987).

[46] L. Manes and A. Barisic, Phys. Status Solidi 3, 971 (1970). 
[47] M. W. Chase, C. A. Davies, J. R. Downey, D. J. Frurip, R. A. McDonald, and A. N. Syverud, Thermochemical Tables 1985 (National Institute of Standards and Technology, Gaithersburg, MD, 1986).

[48] I. J. Fritz, J. Appl. Phys. 47, 4353 (1976).

[49] D. Prieur, P. M. Martin, A. Jankowiak, E. Gavilan, A. C. Scheinost, N. Herlet, P. Dehaudt, and P. Blanchart, Inorg. Chem. 50, 12437 (2011).

[50] F. Lebreton, Synthèse et caractérisation d'oxydes mixtes d'uranium et d'américium, Ph.D. thesis, Université de Limoge, 2014.
[51] C. Thiriet and R. M. J. Konings, J. Nucl. Mater. 320, 292 (2003).

[52] C. Sari and E. Zamorani, J. Nucl. Mater. 37, 324 (1970).

[53] E. Epifano, C. Guéneau, R. C. Belin, R. Vauchy, F. Lebreton, J.-C. Richaud, A. Joly, C. Valot, and P. M. Martin, Inorg. Chem. 56, 7416 (2017).

[54] P. Gotcu-Freis, J.-Y. Colle, C. Guéneau, N. Dupin, B. Sundman, and R. Konings, J. Nucl. Mater. 414, 408 (2011).

[55] H. L. Lukas, S. G. Fries, and B. Sundman, Computational Thermodynamics: The CALPHAD Method (Cambridge University, New York, 2007). 